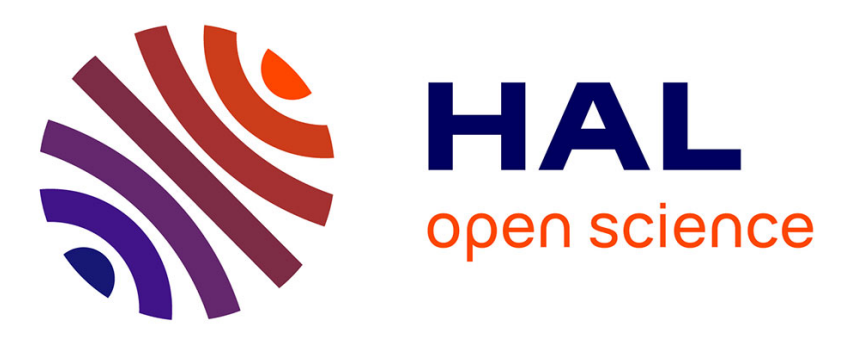

\title{
Analysis of stability and transparency for nanoscale force feedback in bilateral coupling
}

Aude Bolopion, Barthélemy Cagneau, Sinan Haliyo, Stéphane Régnier

\section{To cite this version:}

Aude Bolopion, Barthélemy Cagneau, Sinan Haliyo, Stéphane Régnier. Analysis of stability and transparency for nanoscale force feedback in bilateral coupling. Journal of Micro-Nano Mechatronics, 2008, 4 (4), pp.145-158. 10.1007/s12213-009-0016-3 . hal-02912160

\section{HAL Id: hal-02912160 https://hal.science/hal-02912160}

Submitted on 5 Aug 2020

HAL is a multi-disciplinary open access archive for the deposit and dissemination of scientific research documents, whether they are published or not. The documents may come from teaching and research institutions in France or abroad, or from public or private research centers.
L'archive ouverte pluridisciplinaire HAL, est destinée au dépôt et à la diffusion de documents scientifiques de niveau recherche, publiés ou non, émanant des établissements d'enseignement et de recherche français ou étrangers, des laboratoires publics ou privés. 


\title{
Analysis of stability and transparency for nanoscale force feedback in bilateral coupling
}

\author{
Aude Bolopion - Barthélemy Cagneau • D. Sinan Haliyo · Stéphane \\ Régnier
}

Received: date / Accepted: date

\begin{abstract}
This paper deals with the problem of finding a compromise between stability and transparency for bilateral haptic control in nanorobotics. While manipulating objects with an AFM, real time visual feedback is not available. Force feedback is used to compensate for this lack of visual information. The structure of the control scheme and the value of the controller gains are critical issues for stability, transparency, and ease of manipulation. Two common control schemes are analyzed for submicron scale interactions. Based on stability and transparency criteria, the influence of each of the controllers' gains is derived. The applications for which the bilateral couplings are best suited, as well as their intrinsic limitations are discussed. The theoretical analysis is validated with an experiment composed of several phases with high dynamic phenomena.
\end{abstract}

Keywords Telenanorobotics · Force feedback · Haptic coupling · Bilateral control · Nanomanipulation

\section{Introduction}

Handling of objects at micro or nanoscales is still a challenge especially due to unavailable real time visual feedback while manipulating objects with an AFM, and the difficulty to design accurate grippers and sensors [1]. Haptics appeared to be an interesting solution to deal with these objects [2], after R.L. Hollis developped

A. Bolopion, B. Cagneau, D.S. Haliyo, S. Régnier Université Pierre et Marie Curie, Paris 06

Institut des Systèmes Intelligents et de Robotique CNRS UMR 7222

4 place Jussieu, 75252 Paris Cedex, France

Tel.: +33.1.46.54.78.12

Fax: +33.1.46.54.72.99

E-mail: \{bolopion,cagneau,haliyo,regnier\}@isir.fr the first system to feel the substrate's topology using a master arm [3].

Specific problems arise while dealing with haptics for nanoscale applications. Indeed, scaling factors are needed to set up a bilateral control. We will note $A_{f}$ (resp. $A_{d}$ ) the force (resp. motion) amplification factor. Based on dimensional analysis, [4] presents a method to select these coefficients in order to minimize the environment's distortion. They should be chosen such that $A_{f}=A_{d}$ for surface dominated interactions and $A_{f}=$ $A_{d}^{2}$ for structurally dominated interactions. However, practical limitations, such as the device workspace or the forces that can be felt by the user, will prevent to use these relations.

Scaling factors, as well as time delayed communications or discretization of signals may lead to instability. Tools to cope with such classical problems have been developped for macroscale systems. They include scattering variables and wave variables to deal with time delayed communications ([5], [6]). Another common approach based on the passivity theory is to use observers to monitor the power flows in the system. Damping is added by controllers to dissipate the excess of energy when needed [7]. These tools designed for macroscales have been applied in nanorobotics. For example, wave variables and passivity controllers have been used in simulated environments ([8], [9]). Recent works, including [10], used $H_{\infty}$ theory to get a robust stability against time delays and scaling factors. [11] uses wave variables to ensure stability and also focuses on transparency degradation.

Indeed, the trade-off between stability and transparency is particulary difficult to deal with in nanorobotics. In [12], the authors highlight the equivalent resultant impedance felt by the user compared to that of the environment. However, it does not deal with sta- 
bility. [13] applies a passivity controller to nanomanipulation system. However, some limitations in terms of transparency are pointed out since the pull-in, which is an active phenomenon, is smoothed by the controller. Special care has to be taken while applying this controller to nanoscale applications.

Transparency and stability are the two criteria suitable to evaluate bilateral couplings' performances. The main idea of this paper is to use them to adapt the couplings from the macroscale to the nanoscale. Reminding that scaling factors strongly influence stability, our work will be based on classical controllers well known for macroscale applications in order to study the impact of such factors. The choice of these bilateral couplings is made according to the available inputs and outputs of the nano environment. Hereafter, we only use proportional and proportional-integral controllers so that an analytical analysis can be carried out, to understand the influence of each gain on the system stability and transparency. All the results will be validated through experiments with high dynamic forces. Compared to [14], a transparency analysis is undertaken, and a comparison between two control schemes is made.

This paper is organized as follows: in section 2, we present the experiments and the experimental setup that we will use to validate the control schemes; then, the most intuitive control scheme, the Direct Force Feedback is presented in section 3 . We will show its characteristics for the particular case of nanorobotics. Section 4 introduces another coupling, the Force-Position control scheme. An analysis of its stability and transparency properties is carried out, and will be used to choose the controller gains. The application fields of these couplings are also discussed.

\section{Experimental setup}

\subsection{Coupling validation protocol}

To compare the performance of the different couplings and the influence of the controller gains, we will perform a one-dimensional manipulation. It consists in approaching the substrate with the probe, applying a force and finally retracting from the substrate. The force applied on the cantilever as a function of the distance from the substrate is depicted in figure 1(a) (arrows indicate the chronological path). The corresponding plot of the force applied on the cantilever as a function of time is given in figure $1(\mathrm{~b})$. That is what the operator should ideally feel.

Several points should be noted:

- Two discontinuous phenomena (referenced as A and $\mathrm{E}$ on the plots), are respectively representative of
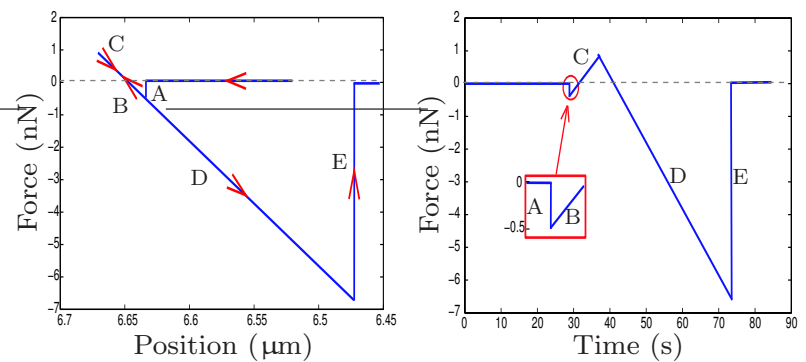

(a) Force as a function of the (b) Force as a function of the position from the substrate time

Fig. 1 Force applied on a cantilever while performing an approach-retract experiment

the instants when the cantilever is attracted (pullin) and released (pull-off) from the substrate.

- Two types of interacting forces are present: the very first (B) and the last (D) are attractive (after the pull-in and before the pull-off), while those in between are repulsive $(\mathrm{C})$.

- The force magnitude variation is high between pullin and pull-off phenomena (factor 10 to 100).

- The pull-in force magnitude is in the order of tenth of nanonewtons.

The bilateral coupling must render to the user these phenomena present at nanoscales. As its structure depends on the used devices, the experimental platform will be detailed before performing any analysis.

\subsection{Experimental platform}

\subsubsection{System devices}
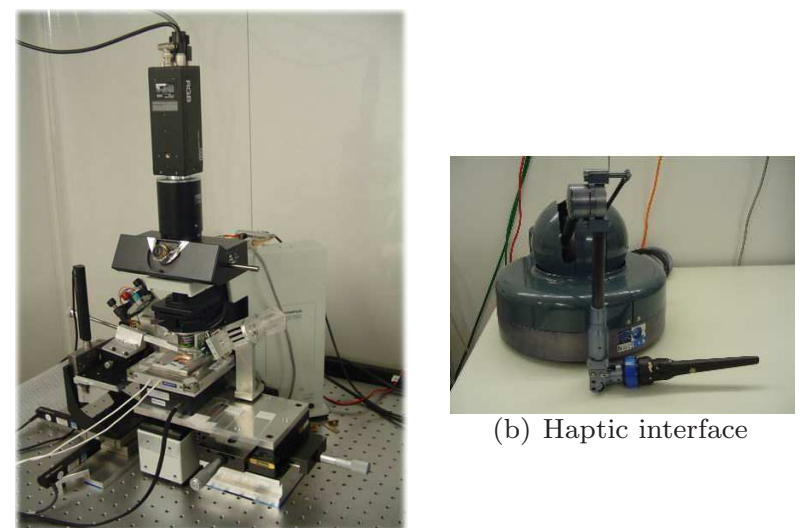

(b) Haptic interface

(a) General view of the platform

Fig. 2 Experimental setup

The experimental platform used to validate the coupling is depicted in figure 2(a). It is composed of a 
nanotranslator (manufactured by Physik Instrumente) ${ }^{1}$ with a motion range of $12 \mu \mathrm{m}$ and a resolution of $1.83 \mathrm{~nm}$. It moves the substrate along the vertical direction, to approach or retract it from a fixed cantilever.

The force applied by the substrate on the cantilever $\left(F_{e}\right)$ is measured with a laser. The deflection of the probe is measured using a beam focused on the cantilever, which is reflected onto a photodiode. [15] gives more details on laser optics. Then the normal force $F_{e}$ can be computed from equation (1):

$F_{e}=k_{c} d=k_{c} S V$

where:

$-k_{c}$ : stiffness of the cantilever (from a hundredth up to several dozen $\mathrm{N} \cdot \mathrm{m}^{-1}$ ) - calibrated as it was demonstrated in [16]. Note that $\mu \mathrm{N} \cdot \mathrm{m}^{-1}$ or $\mathrm{nN} \cdot \mathrm{m}^{-1}$ are also consistent units, more adapted to describing nanoscale phenomena

$-d$ : deflection of the cantilever

- $S$ : sensitivity of the calibrated photodiode

- $V$ : measured output voltage

As highlighted in equation (1), the force depends on the stiffness of the cantilever. This parameter may vary significantly depending on the performed task. Manipulation operations require stiff probes to interact precisely with objects, whereas soft ones can measure smaller forces. Therefore they are suited for exploration or teaching purposes. The bilateral coupling analysis should take into account the cantilever stiffness.

The haptic device used is a 3 degrees of freedom of force feedback Virtuose, manufactured by Haption ${ }^{2}$ (figure 2(b)).

\subsubsection{Power flow}

Figure 3 summarizes the power flow between the different subsystems (operator, haptic interface, coupling, slave device and environment), and the inputs and outputs of the subsystems. $F_{m}$ represents the force fed back to the user, while $V_{s}$ is the desired velocity set to the nanotranslator.

The bilateral coupling must be designed accordingly to the subsystem's inputs and outputs (master and slave devices' characteristics, sensors available in the environment). However, it is not really restrictive since many teleoperation systems for nanoscale applications presented in the literature fulfill these requirements ([17], [18], [19]).

\footnotetext{
1 http://www.physikinstrumente.com/

2 http://www.haption.com/
}

\subsection{Objectives}

The study of the haptic coupling properties will be based on the classical theory of automation. Our objective is to determine the influence of the different controller gains on the coupling performances. We will use stability criteria, as Routh-Hurwitz or Llewelyn, and Bode analysis of the transfer functions. The second step will be to find the parameters that will lead to the most transparent, but still stable, control scheme. This will be done according to the conclusions about the influence of each gain on the system. This proposed tuning must be robust with respect to the environment, and especially the cantilever's stiffness. Then we will be able to determine the applications for which the control schemes are the best suited, considering their intrinsic structure and properties. All these conclusions will be validated by experiments based on the protocol described in paragraph 2.1 .

\section{Direct Force Feedback}

In this section the first control scheme, namely Direct Force Feedback ( $D F F)$ is introduced and analysed. Stability (using the Routh-Hurwitz criterion) and transparency issues are considered to derive its specificities concerning nanoscale applications. Approach-retract experiments are conducted using different control parameters to verify the analysis.

\subsection{Control scheme structure}

This control scheme, depicted in figure 4 , is the most intuitive formulation to provide amplified forces to the operator [20]. Basically, the user operates a haptic device in the macro-world to impose the displacements of the slave device in the nano-world. The blocks and power flows defined in figure 3 are clearly identified. The controller scales down the motions provided by the user by a coefficient $A_{d}$, and magnifies environmental forces by a factor $A_{f}$ to provide force feedback.

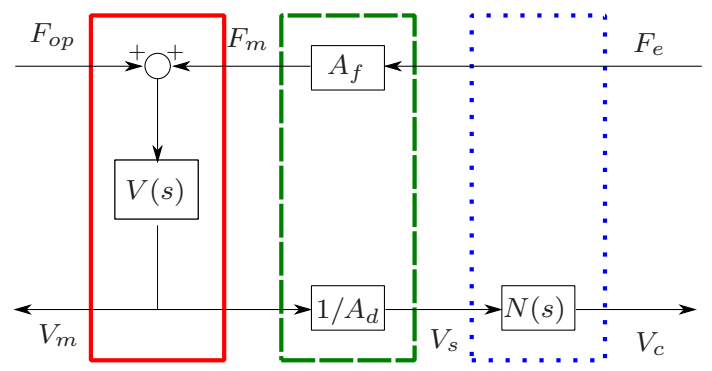

Fig. 4 Direct Force Feedback control scheme 


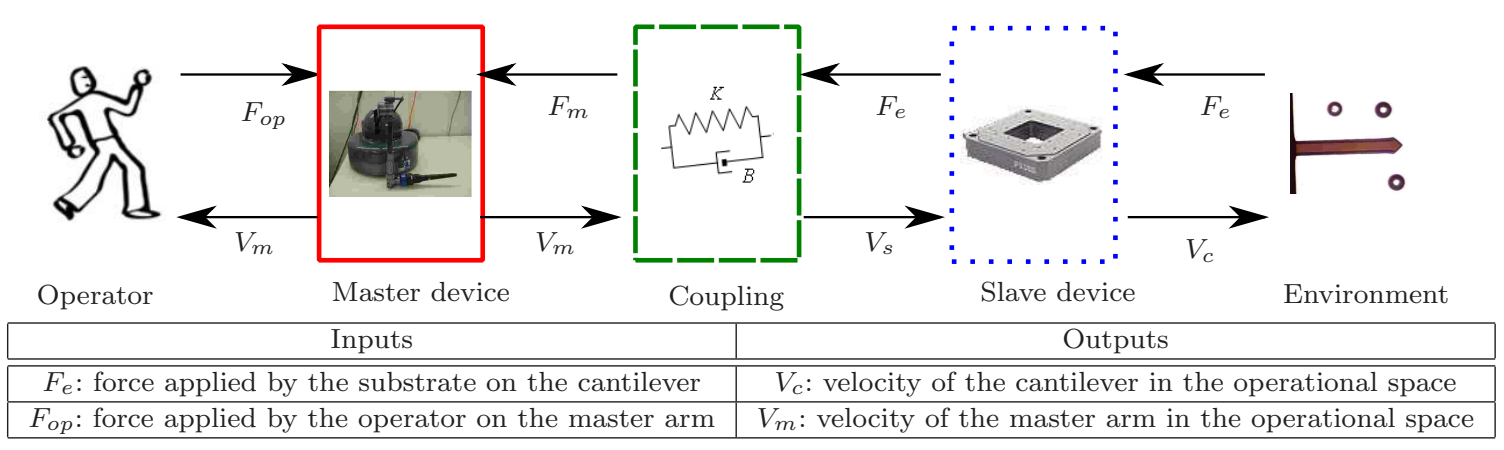

Fig. 3 Power flow between the subsystems

The master arm is modeled by a rigid body for which inertia and damping are respectively $M_{v}$ and $B_{v}$. The slave robot's transfer function is modeled by a second order function with two time constants $\tau_{1}$ and $\tau_{2}$ :

$$
\begin{aligned}
& V(s)=\left[\left(B_{v}+M_{v} s\right)\right]^{-1} \\
& N(s)=\left[\left(1+\tau_{1} s\right)\left(1+\tau_{2} s\right)\right]^{-1}
\end{aligned}
$$

where $s$ is the Laplace variable. Numerical values of the parameters have been identified as:

$$
\begin{aligned}
& M_{v}=0.4 \mathrm{~kg} \quad ; B_{v}=0.1 \mathrm{~N} \cdot \mathrm{s} \cdot \mathrm{m}^{-1} \\
& \tau_{1}=1.35 \cdot 10^{-3} \mathrm{~s} ; \tau_{2}=0.57 \cdot 10^{-3} \mathrm{~s}
\end{aligned}
$$

\subsection{Stability}

The slave device interacts with a remote environment, which must be considered for the stability analysis. Two approaches can be used. The first is to consider a method that is applicable for any passive environments (e.g., methods based on passivity analysis). However, they are more conservative since stability is guaranteed for any environment as long as it is passive. Therefore, they are not useful for our concern to point out the limitations induced by the cantilever's stiffness or the environment's characteristics.

The second approach is to model the environment and to consider stability with respect to the specificities of both the coupling and the environment. It will be shown theoretically and experimentally that this control scheme is stable in a given context. To model the environment, assumption (1) is made:

Assumption 1 The slave device is linked with its environment through a spring constant of stiffness $k_{c}$ (the cantilever). The substrate is modeled as a spring $k_{s}$. These two serial springs are linked such that the equivalent stiffness $k_{e q}$ is:

$\frac{1}{k_{e q}}=\frac{1}{k_{s}}+\frac{1}{k_{c}}$
Hertz's theory is widely used to model the contact between a cantilever and the substrate [21]. In the case of a contact between a sphere (which can be the extremity of a cantilever's tip) and a plane, it states that the contact stiffness $k_{s}$ is:

$k_{s}=\frac{3}{2} K a$

The variables are listed below, numerical values are given for a silicon cantilever and a glass substrate:

- $a$ : contact area between the sphere and the plane $a^{3}=\frac{R_{t} F_{e}}{K}$

- $K$ : equivalent Young's modulus of the sphere and the plane $K=\frac{1}{\frac{3}{4}\left(\frac{1-\nu_{1}^{2}}{E_{1}}+\frac{1-\nu_{2}^{2}}{E_{2}}\right)}$

- $E_{1,2}$ : Young's modulus for the cantilever and substrate (respectively $E_{1}=150 \mathrm{GPa}$ and $E_{2}=69 \mathrm{GPa}$ )

$-\nu_{1,2}$ : Poisson's ratio for the cantilever and substrate (respectively $\nu_{1}=0.17$ and $\nu_{2}=0.25$ )

- $R_{t}$ : sphere's radius of curvature $R_{t}=10 \mathrm{~nm}$

Even if the assumption (1) is not very restrictive, it is sufficient to point out the inherent problems of the proposed control scheme in the nano-world. Considering this assumption, and the control scheme depicted in figure 4 , the transfer function between $F_{o p}$ and $V_{m}$ can be derived. Since the system is linear-time invariant (LTI), the Routh-Hurwitz criterion can be applied to this transfer function. A necessary and sufficient condition of stability is:

$R=\frac{A_{f}}{A_{d}}<\frac{\gamma}{k_{e q}}=R_{\text {max }}$

where $\gamma=\frac{B_{v}\left(\tau_{1}+\tau_{2}\right)\left[M_{v}^{2}+M_{v} B_{v}\left(\tau_{1}+\tau_{2}\right)+B_{v}^{2} \tau_{1} \tau_{2}\right]}{\left[M_{v}\left(\tau_{1}+\tau_{2}\right)+B_{v} \tau_{1} \tau_{2}\right]^{2}}$

As $\gamma$ only depends on the systems' parameters, for a given environment, according to equation (6) the system's stability only depends on the ratio $\frac{A_{f}}{A_{d}}$.

The worst case for the issue of stability is when the equivalent stiffness is the highest. Using equation (4), this corresponds to $k_{e q}=k_{c}$. In the following sections we will use this approximation. 


\subsection{Transparency}

Transparency of haptic couplings is defined in [22], [23]. It is based on the comparison between the impedance of the environment $Z_{e}=-F_{e} / V_{c}$ and that felt by the operator $Z_{o p}=F_{o p} / V_{m}$. Ideal transparency is achieved when:

$Z_{o p}=Z_{e}$

However, for submicron scales, equation (7) does not make any sense. It is necessary to consider the scaling factors $A_{f}$ and $A_{d}$ such that the impedances can be compared. In our context, perfect transparency will be achieved if:

$\frac{F_{o p}}{V_{m}}=\frac{-A_{f} F_{e}}{A_{d} V_{c}} \Leftrightarrow Z_{o p}=\frac{A_{f}}{A_{d}} Z_{e}$

It has to be noted that the force sensor used modifies the profile of the measured forces, and therefore the operator's feeling. However, we will not deal with that issue, as we will focus only on the influence of the coupling and the haptic device.

Using the control scheme depicted in figure 4, the impedance felt by the operator is derived:

$Z_{o p}=\frac{F_{o p}}{V_{m}}=\frac{A_{f}}{A_{d}} Z_{e} N(s)+\frac{1}{V(s)}$

\subsubsection{Contact}

While in contact, the impedance felt by the operator will be that of equation (9). According to equation (8), this corresponds to the impedance he should ideally feel $\left(\frac{A_{f}}{A_{d}} Z_{e}\right)$ modulated by the nanotranslator dynamic. It is also influenced by the haptic device characteristics.

In the frequency domain $(s=j \omega)$, equation (9) can be rewritten as:

$Z_{o p}=\frac{A_{f}}{A_{d}} Z_{e} \frac{1}{\tau_{1} \tau_{2} \omega^{2}+\left(\tau_{1}+\tau_{2}\right) j \omega+1}+\left(M_{v} j \omega+B_{v}\right)$

For low frequencies, the impedance $Z_{o p, L F}^{D F F}$ can be approximated by:

$Z_{o p, L F}^{D F F} \underset{\omega<<1}{\approx} \frac{A_{f}}{A_{d}} Z_{e}+B_{v}$

The user feels the environmental impedance, as well as the viscosity of the haptic interface. However, $B_{v}$ can be set aside compared to $\frac{A_{f}}{A_{d}} Z_{e}$.

For high frequencies, the impedance $Z_{o p, H F}^{D F F}$ is:

$Z_{o p, H F}^{D F F} \underset{\omega>>1}{\approx} M_{v} j \omega$
This result is valid as far as the stiffness of $Z_{e}$ is a finite value. Since $Z_{e}$ can be computed as $Z_{e}=\frac{k_{e q}}{j \omega}$, and according to assumption (1) $k_{e q}=k_{c}, Z_{e} \underset{\omega>>1}{\approx} 0$.

According to equation (12), the transparency of the coupling is only affected by the inertia of the haptic device for high frequencies.

The Bode's diagram corresponding to these contact impedances is plotted in figure 5 .

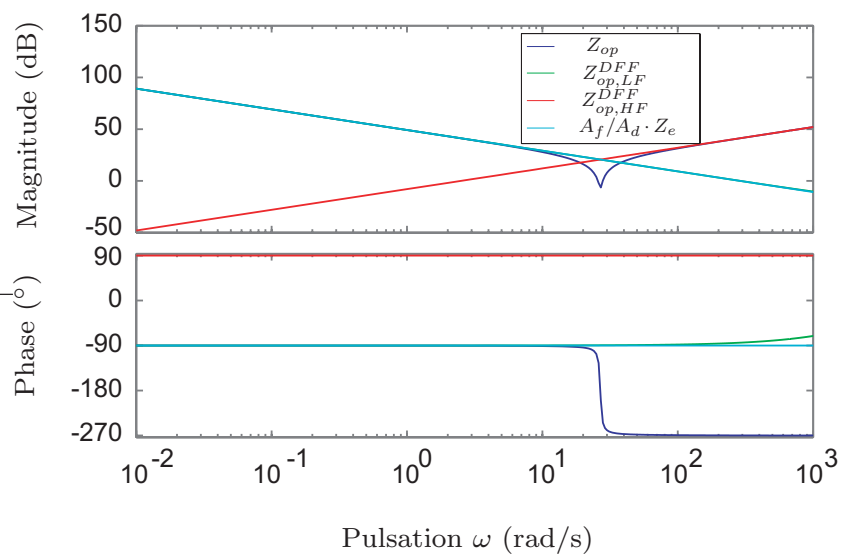

Fig. 5 Bode's diagram for contact impedances, $D F F$ control scheme

Remark 1 To plot figure 5 and following Bode's diagrams, the values $k_{e q}=2.4 \mathrm{~N} \cdot \mathrm{m}^{-1}, A_{f}=6 \cdot 10^{6}$ and $A_{d}=0.05 \cdot 10^{6}$ (these values will be used to perform experiments in the following sections). However, the same conclusions would have been derived for any values of these parameters.

Figure 5 confirms that the system is perfectly transparent for low frequencies (and the viscosity of the haptic interface can indeed be set aside), and affected by the haptic device's inertia for high frequencies.

\subsubsection{Non-contact}

When no forces are applied to the cantilever, the environment's impedance is $Z_{e}=0$, which, according to equation (10), leads to:

$Z_{o p}=M_{v} j \omega+B_{v}$

At low frequencies, the operator will mainly feel the viscosity of the haptic device. At high frequencies, the inertia of the master arm will be predominant. This can indeed be verified in the Bode's diagram depicted in figure 6 .

Using the DFF control scheme, the rendering will only depend on the haptic device characteristics, for both contact and non contact modes. 


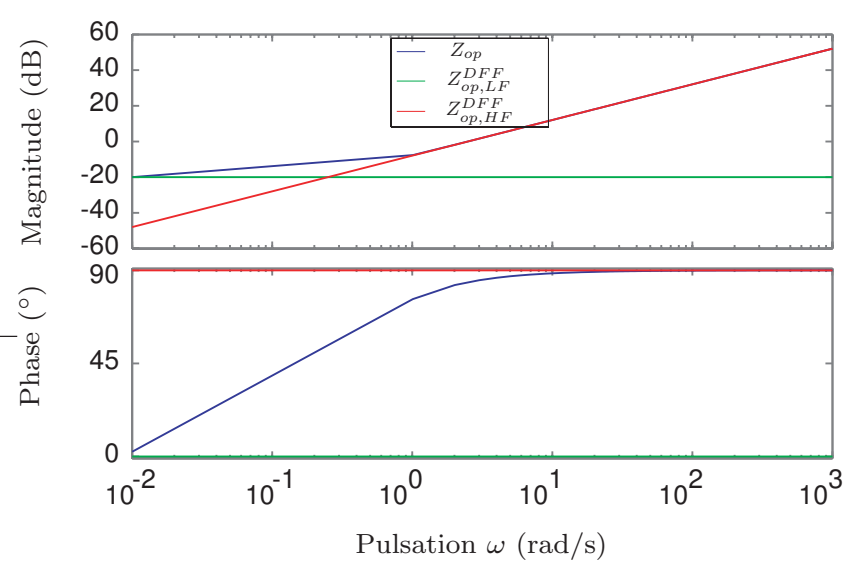

Fig. 6 Bode's diagram for non contact impedances, $D F F$ control scheme

\subsection{Determination of the control scheme parameters}

As seen in the previous stability and transparency analyses, the control scheme parameters determines the performances of the system. Based on the previous paragraph conclusions, this section will highlight the influence of the gains on the coupling. For this control scheme, only two parameters must be tuned: $A_{f}$ and $A_{d}$. As seen in section 3.3, the system transparency only depends on the master and slave devices, but not on the gain values. Therefore, only stability considerations must be considered. Figure 7(a) allows the user to select $A_{f}$ and $A_{d}$ taking into account the equation (6), for a particular value of $k_{e q}$. The figure $7(\mathrm{~b})$ represents the region of stability for different values of $k_{e q}$. As the stiffness increases, the condition of stability becomes stricter.
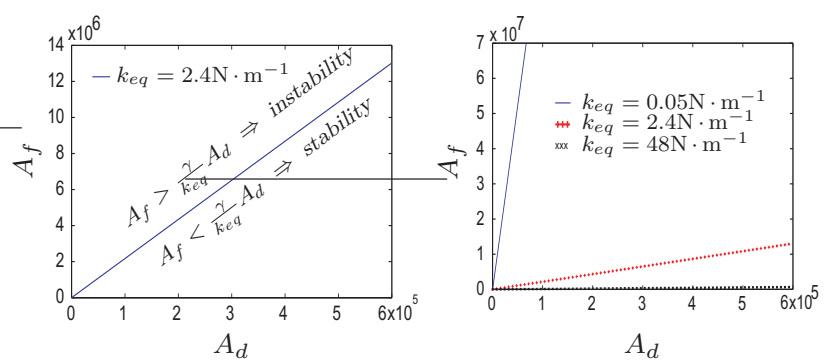

(a) Stability and instability re- (b) Evolution of the stability gions for $k_{e q}=2.4 \mathrm{~N} \cdot \mathrm{m}^{-1} \quad$ and instability regions for different $k_{e q}$

Fig. 7 System's stability with respect to $A_{f}$ and $A_{d}$.

It should also be noted that $A_{f}$ and $A_{d}$ are scaling factors, and must also be chosen according to amplification requirements.

\subsection{Experimental results}

\subsubsection{Contact and pull-off forces}

A cantilever with a spring constant $k_{c}=2.4 \mathrm{~N} \cdot \mathrm{m}^{-1}$ is used. According to equation (6) and figure 7(a), the maximum value allowed for the ratio $R$ to assure a stable system is:

$R_{\max }=21.7$

$A_{d}$ is chosen according to the master and slave motion ranges. To get a good compromise between easiness and accuracy of manipulation, the value $A_{d}=0.05 \cdot 10^{6}$ is selected (the master motion range corresponds then to a displacement of $5 \mu \mathrm{m}$ for the slave). $A_{f}$ is selected so that the system remains stable, i.e. so that the RouthHurwitz criterion given in equation (6) is satisfied. The value $A_{f}=0.12 \cdot 10^{6}$ will be used.

The obtained results (forces sent back to the user) are depicted in figure 8. During the first stage (C), the user applies forces on the substrate (the maximum is $0.30 \mathrm{~N})$. While retracting, the user must counterbalance the forces resulting from the adhesion effects (D). A force equal to $-0.13 \mathrm{~N}$ is necessary to release the cantilever from the substrate (E).

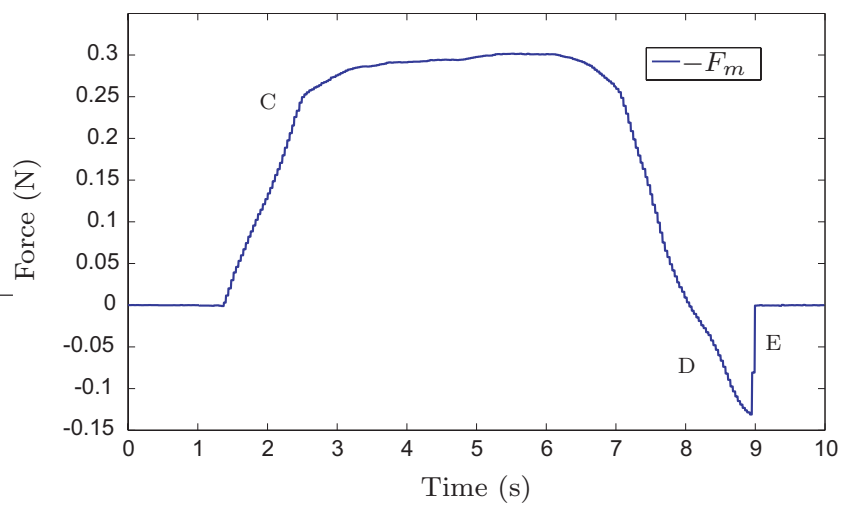

Fig. 8 Approach-retract curve for a cantilever of stiffness $k_{c}=$ $2.4 \mathrm{~N} \cdot \mathrm{m}^{-1}$, using $A_{d}=0.05 \cdot 10^{6}, A_{f}=0.12 \cdot 10^{6} . R<R_{\max }$. $D F F$ control scheme.

The system remains stable and the forces sent back to the user are equal to those measured by the photodiode scaled by $A_{f}\left(F_{m}=A_{f} F_{e}\right.$ according to the control scheme's design). Therefore, the operator indeed feels phenomena happening in the environment. However, using our haptic interface, forces fed back remain too low to increase the accuracy of the performed task. The force scaling factor has to be increased in order to provide better force feedback.

During this second experiment, the value of $A_{d}$ remained the same, but $A_{f}$ was increased: $A_{f}=6.0 \cdot 10^{6}$. 
With these values:

$R=120>R_{\max }$

The Routh-Hurwitz criterion is no longer satisfied. Consequently, the system is predicted to be unstable. The results are plotted in figure 9 .

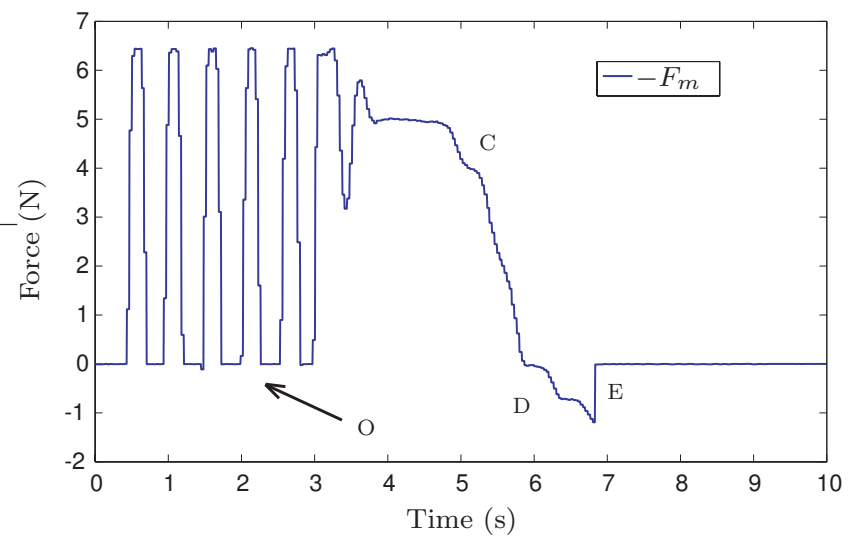

Fig. 9 Approach-retract curve for a cantilever of stiffness $k_{c}=$ $2.4 \mathrm{~N} \cdot \mathrm{m}^{-1}$, using $A_{d}=0.05 \cdot 10^{6}, A_{f}=6 \cdot 10^{6} . R>R_{\max } . D F F$ control scheme.

Remarkably, the forces have been amplified compared to the forces in figure 8. It is then easier for the user to detect repulsive and attractive forces $(\mathrm{C}, \mathrm{D})$ and the high variation of the forces due to the pulloff (E). Although force reflection has been improved by modifying the value of $A_{f}$, the system's stability is affected. When the cantilever establishes contact with the substrate, it creates high amplitude oscillations in the system $(\mathrm{O})$. This is very disturbing for the user who has to act like a damper to absorb the excessive energy responsible for the instability.

For a given velocity-scaling factor, the $D F F$ control scheme suffers from a trade-off between stability and force amplification.

\subsubsection{Pull-in, contact and pull-off forces}

To render the pull-in force to the user, it is necessary to increase $A_{f}$. However, as seen in equation (6) and demonstrated in figure 9 , if $A_{d}$ is kept constant, the system is unstable. Thus, it should be increased to ensure stability. Since $A_{d}$ represents the velocity-scaling factor, this implies that the user will need to move the haptic handle over longer distances than in the experiment depicted in figure 8 to perform the same displacement. This makes the experiment very long, as it will be demonstrated later.
According to equation (6), the cantilever stiffness must also be considered for stability. Since $F_{m}=A_{f} F_{e}$ and $F_{e}=k_{c} d$ where $d$ is the cantilever deflection, equation (6) becomes:

$A_{d} \geq \frac{1}{d} \frac{F_{m}}{\gamma}$

For the same value $F_{m}$ fed back to the user, $A_{d}$ is proportional to the inverse of the deflection. Considering the pull-in phenomenon, the deflection of soft cantilevers is bigger than that of stiff ones. Consequently, the jump into contact will happen when the probe is higher from the substrate. Therefore, the condition on $A_{d}$ to assure stability is stricter for stiff cantilevers. We will therefore choose a softer cantilever $\left(k_{c}=0.05 \mathrm{~N} \cdot \mathrm{m}^{-1}\right)$.

To be able to feel the pull-in force, we chose $A_{f}=$ $6700 \cdot 10^{6}$ and $A_{d}=50 \cdot 10^{6}$. For this cantilever, $R_{\max }=$ 1040. Therefore, for these scaling factors, the system is predicted to be stable.

The results obtained are presented in figure 10 . The forces that should have been sent back to the user, as well as those actually felt by the operator are shown (i.e., after saturation). As previously explained, the pullin phenomenon is between 10 to 100 times weaker than the pull-off. Therefore, if the scaling factors are kept constant, the force corresponding to the pull-off is strongly amplified and neither the haptic device nor the operator can cope with such forces. That is why forces are saturated to $5 \mathrm{~N}$, so that the manipulation remains comfortable for the user. The pull-in effect can clearly be felt since the peak amplitude is around $2 \mathrm{~N}$.

However, since $A_{d}$ has been increased in order to keep the system stable, the experiment is time consuming (one and a half minutes whereas the cantilever was soft and initially set very close to the contact point).

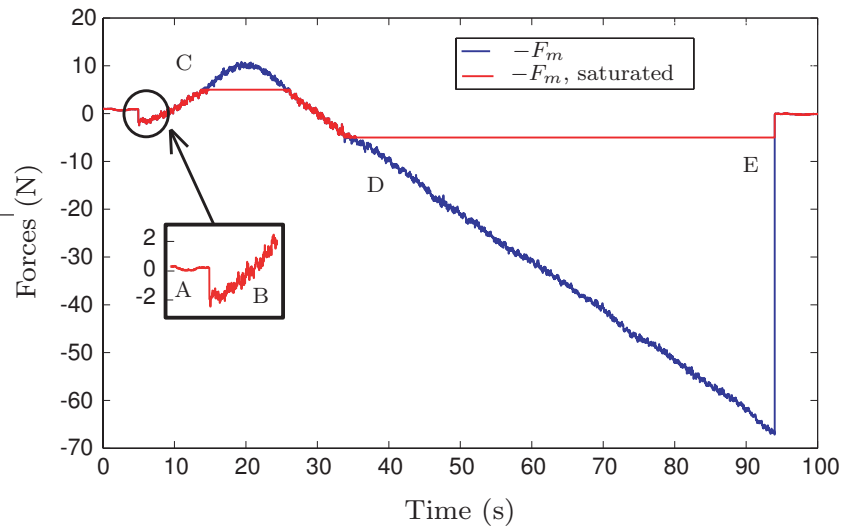

Fig. 10 Approach-retract curve with pull-in for a cantilever of stiffness $k_{c}=0.05 \mathrm{~N} \cdot \mathrm{m}^{-1}, A_{d}=50 \cdot 10^{6}, A_{f}=6700 \cdot 10^{6} . R<$ $R_{\max }$. DFF control scheme. 
The $D F F$ control scheme is highly transparent, since both pull-in and pull-off phenomena were felt. However, it suffers from a trade-off between force amplification and velocity-scaling, which can result either in instabilities or time consuming experiments. Therefore, for easier manipulations, it is necessary to modify the structure of the control scheme to add some damping on the system. This can avoid instabilities that appear in figure 9 while reaching the contact.

In section 4.1, a second well-known control scheme, which takes into account these necessary conditions to avoid the trade-off pointed out above, will be compared to the DFF in the special context of micro and nanorobotics.

\section{Force-Position control}

In this section, the Force-Position ( FP) control scheme is studied. Stability analysis is conducted using discrete time variable $z$ since it involves numerical integrations which make the system very sensitive to the sampling period. Transparency analysis is carried out in the continuous-time domain in order to compare the results with those obtained for the DFF control scheme. Based on the conclusions, we will choose the gains of the FP controller, and we will define what the applications of such a coupling are.

\subsection{Control scheme structure}

As for the $D F F$, the inputs of the Force-Position control scheme are the velocity of the haptic device handle and the force applied by the environment on the cantilever (figure 11). The outputs are the velocity used as the

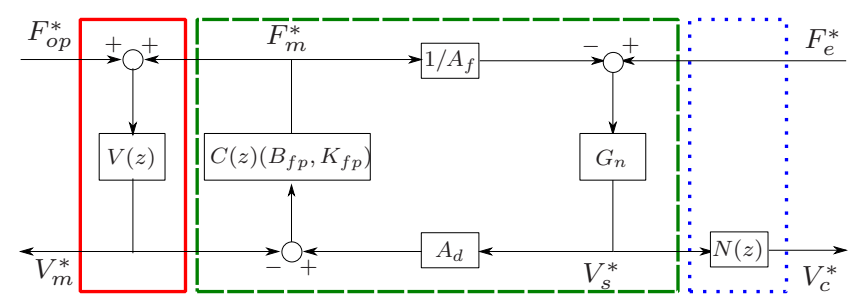

Fig. 11 Force-Position control scheme

desired reference for the nanotranslator and the force that will be fed back to the user by the haptic device. As previously, $A_{f}$ and $A_{d}$ are respectively the force and velocity scaling factors.

Discrete time formulation is used to take into account effects of the sampling period $T_{e} . z$ represents the discrete time variable. The star superscript is for discrete parameters. It was shown in section 3 that the $D F F$ control scheme presents limitations in terms of stability, depending on the desired scaling factors. To improve this issue, two controllers are added in the FP coupling. $G_{n}$ and $C(z)$ are respectively a proportional $(P)$ and a proportional-integral $(P I)$ controller. $C(z)$ has been discretized using Tustin's approximation:

$C(z)=B_{f p}+K_{f p} \frac{T_{e}(z+1)}{2(z-1)}$

In the following, $V(z)$ (resp. $N(z)$ ) will refer to discrete time transfer functions corresponding to $V(s)$ (resp. $N(s))$. They are computed using the Z-transform function $Z\{$.$\} :$

$V(z)=\left(1-z^{-1}\right) Z\left\{\frac{V(s)}{s}\right\}=\frac{1}{B_{v}} \frac{1-\delta}{z-\delta}$

where $\delta=e^{-\frac{B_{v} T_{e}}{M_{v}}}$.

$N(z)=1-\alpha_{1} \frac{z-1}{z-e^{-\frac{T_{e}}{\tau_{1}}}}-\alpha_{2} \frac{z-1}{z-e^{-\frac{T_{e}}{\tau_{2}}}}$

where $\alpha_{1}=\frac{1}{1-\frac{\tau_{2}}{\tau_{1}}}$ and $\alpha_{2}=\frac{1}{1-\frac{\tau_{1}}{\tau_{2}}}$.

Compared to the DFF control scheme, the feedback force $F_{m}$, is computed with the $P I$ controller. The integral gain $K_{f p}$ and the proportional gain $B_{f p}$ can be used to modify the stiffness and damping of the rendered force. The gain $G_{n}$ is used to compute the desired velocity of the slave device.

\subsection{Stability}

To derive the stability conditions for the FP control scheme, a first approach is to verify the Routh-Hurwitz criterion. This method was previously used in section 3. However, it has been noticed that the environment must be modeled. Considering that three gains and two scaling factors are used in the control scheme depicted in figure 11, it is obvious that the relationships between these parameters will be complex and will not allow to highlight the influence of each of the gains on stability. Therefore, the Routh-Hurwitz criterion is not appropriated to analyze the stability of this bilateral coupling. To assure the system's stability without modeling the environment, two main approaches are currently used. The first is passivity [7], which deals with energy flow in the system. The second is absolute stability [24]. As for passivity, it ensures that if the control scheme is connected to passive blocks (in our case the environment and the operator which can be considered passive as in [25]), the system will remain stable. Both of these criteria lead to sufficient but not necessary conditions. 
Since absolute stability is less conservative, we will use this criterion, based on Llewelyn's theorem.

The admittance matrix $\mathbf{P}$ of the coupling is defined as:

$$
\begin{aligned}
{\left[\begin{array}{c}
V_{m}^{*} \\
V_{c}^{*}
\end{array}\right] } & =\mathbf{P}(z) \cdot\left[\begin{array}{c}
F_{o p}^{*} \\
F_{e}^{*}
\end{array}\right] \\
& =\left[\begin{array}{l}
p_{11}(z) p_{12}(z) \\
p_{21}(z) p_{22}(z)
\end{array}\right] \cdot\left[\begin{array}{c}
F_{o p}^{*} \\
F_{e}^{*}
\end{array}\right]
\end{aligned}
$$

where:

$$
\begin{aligned}
& p_{11}(z)=V(z)\left[A_{f}+A_{d} G_{n} C(z)\right] / D(z) \\
& p_{12}(z)=\left[A_{f} A_{d} G_{n} C(z) V(z)\right] / D(z) \\
& p_{21}(z)=\left[G_{n} V(z) C(z)\right] / D(z) \\
& p_{22}(z)=A_{f} G_{n}[1+C(z) V(z)] / D(z) \\
& D(z)=A_{f}+A_{d} G_{n} C(z)+A_{f} V(z) C(z)
\end{aligned}
$$

\section{Theorem 1 (Llewelyn [26])}

A system represented by the admittance matrix $\mathbf{P}$ is unconditionally stable if and only if the following conditions hold:

$$
\begin{aligned}
C_{1}=\operatorname{Re}\left(p_{11}\right) & \geq 0 \\
C_{2}=\operatorname{Re}\left(p_{22}\right) & \geq 0 \\
C_{3}=2 \operatorname{Re}\left(p_{11}\right) \operatorname{Re}\left(p_{22}\right)-\left|p_{12} p_{21}\right|-\operatorname{Re}\left(p_{12} p_{21}\right) & \geq 0
\end{aligned}
$$

These inequalities will be used to verify if the chosen gains meet stability requirements.

\subsection{Transparency}

In order to compare the results to those obtained for the $D F F$, transparency was studied in the continuous time domain. Using the control scheme depicted in figure 11, the impedance felt by the operator $Z_{o p}=F_{o p} / V_{m}$ can be computed:

$Z_{o p}=\frac{n_{z_{e}} Z_{e}+A_{f} C(s) V(s)+A_{f}+A_{d} G_{n} C(s)}{d_{z_{e}} Z_{e}+A_{f} V(s)+A_{d} G_{n} C(s) V(s)}$

where:

$$
\begin{aligned}
& n_{z_{e}}=A_{f} G_{n} C(s) V(s) N(s)+A_{f} G_{n} N(s) \\
& d_{z_{e}}=A_{f} G_{n} V(s) N(s)
\end{aligned}
$$

\subsubsection{Contact}

When the contact is established, $Z_{o p, L F}^{F P}$ can be computed for low frequencies:

$Z_{o p, L F}^{F P} \underset{\omega<<1}{\approx} \frac{A_{f}}{A_{d}+\frac{A_{f} k_{e q}}{K_{f p}}} Z_{e}$
As $K_{f p}$ increases, the impedance felt by the operator tends to the ideal impedance $\frac{A_{f}}{A_{d}} Z_{e}$.

For high frequencies, the impedance $Z_{o p, H F}^{F P}$ felt by the user is:

$Z_{o p, H F}^{F P} \underset{\omega>>1}{\approx} M_{v} j \omega$

For the same reasons as the ones exposed in section 3.3.1, the user only feels the inertia of the haptic device for $\omega>>1$.

Bode's diagram represented in figure 12 is useful to illustrate the analytical results, and to compare them to those obtained for the DFF control scheme.

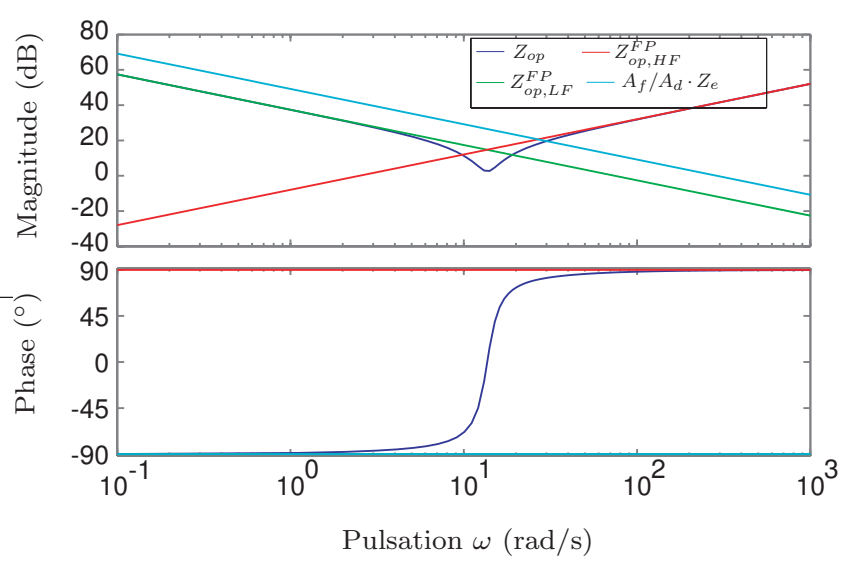

Fig. 12 Bode's diagram for contact impedances, FP control scheme

Remark 2 Bode's diagram (figure 12), and followings have been plotted for specific values: $k_{e q}=2.4 \mathrm{~N} \cdot \mathrm{m}^{-1}$, $A_{f}=6 \cdot 10^{6}, A_{d}=0.05 \cdot 10^{6}$ (the same values as for figure 5$), K_{f p}=100 \mathrm{~N} \cdot \mathrm{m}^{-1}, B_{f p}=2 \mathrm{~N} \cdot \mathrm{s} \cdot \mathrm{m}^{-1}$ and $G_{n}=48.0 \mathrm{~m} \cdot \mathrm{N}^{-1} \cdot \mathrm{s}^{-1}$. As already underscored in remark 1, these values are only defined to illustrate the commentary, but do not change the conclusions.

For low frequencies, the variations in the Bode's magnitude are the same for the simulated contact impedance and for the environment. The real and felt impedances only differ by a static gain which can be reduced by increasing $K_{f p}$. Consequently, when the user reaches the contact point, he is able to detect the variations of the forces involved during the process. Although the system is not as perfectly transparent as $D F F$, it is well suited for manipulation tasks. 


\subsubsection{Non-contact}

When no force is acting on the cantilever, the operator feels the impedance:

$Z_{o p}=\frac{A_{f}\left(K_{f p}+B_{f p} j \omega\right)}{A_{f} j \omega+A_{d} G_{n}\left(K_{f p}+B_{f p} j \omega\right)}+M_{v} j \omega+B_{v}$

For low frequencies, the impedance $Z_{o p, L F}^{F P}$ can be approximated from:

$Z_{o p, L F}^{F P} \underset{\omega<<1}{\approx} \frac{A_{f}}{A_{d} G_{n}}+B_{v}$

To minimize the impedance felt by the operator, without affecting the scaling factors, $G_{n}$ is the only parameter that can be tuned. The higher it is, the better the transparency will be. However, whatever the values of the control scheme's gains, the user will feel the viscosity of the haptic interface (as was the case for the $D F F$ control scheme).

At higher frequencies, he feels the impedance $Z_{o p, H F}^{F P}$

$Z_{o p, H F}^{F P} \underset{\omega>>1}{\approx} M_{v} j \omega$

As for the $D F F$, the operator will feel the inertia of the haptic device.

The Bode's diagram (figure 13) confirms the validity of the approximations made in equations (30) and (31). When compared to the Bode's diagram for the DFF control scheme (figure 6) for non-contact, it highlights the lack of transparency for low frequencies. However, this difference can be reduced by increasing $G_{n}$.

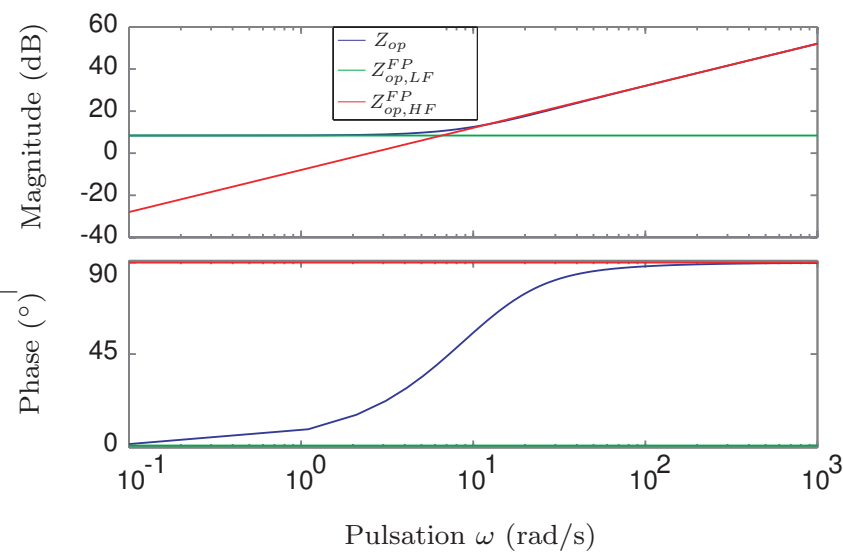

Fig. 13 Bode's diagram for non contact impedances, FP control scheme

As highlighted in table 1 which summarizes the approximated impedance for the $D F F$ and $F P$ control schemes, the FP coupling is less transparent than the
Table 1 Approximated values of $F_{o p}$ for $D F F$ and $F P$ control schemes

\begin{tabular}{|c|c|c|c|}
\hline & & $D F F$ & $F P$ \\
\hline \multirow{2}{*}{ 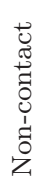 } & low frequencies & $B_{v}$ & $\frac{A_{f}}{A_{d} G_{n}}+B_{v}$ \\
\hline & high frequencies & $M_{v} j \omega$ & $M_{v} j \omega$ \\
\hline \multirow{2}{*}{ 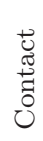 } & low frequencies & $\frac{A_{f}}{A_{d}} Z_{e}+B_{v}$ & $\frac{A_{f} K_{f p}}{A_{d} K_{f p}+A_{f} k_{e q}} Z_{e}$ \\
\hline & high frequencies & $M_{v} j \omega$ & $M_{v} j \omega$ \\
\hline
\end{tabular}

$D F F$. To obtain the same transparency $K_{f p}$ has to be high enough to ensure a stiff contact, while $G_{n}$ will influence the non-contact behavior of the coupling. The higher it is, the less viscous the feeling for the operator will be.

\subsection{Determination of the control scheme parameters}

The performance of this bilateral coupling highly depends on the controller parameters and the scaling factors (see paragraphs 4.2 and 4.3). However, relations derived in the previous sections do not allow to easily choose the gains since each of them is composed of many parameters. It will be useful to consider particular cases to derive simple necessary conditions of stability. Using these relations, and transparency considerations, gains will be chosen. Then, Llewelyn criterion will be used to see if the gains meet sufficient stability conditions.

\subsubsection{Scaling factors}

As in the first control scheme, $A_{f}$ and $A_{d}$ depend on user's requirements. Indeed, one might want a precise positioning, and/or important force feedback, according to the manipulation tasks. As in section 3, we will choose $A_{d}=0.05 \cdot 10^{6}$. $A_{f}$ will be such that the forces sent back to the user are high enough for an untrained user to distinguish the different phenomena encountered during the experiment.

\subsubsection{Proportional controller $G_{n}$}

Problems of stability can be due to control schemes but also to numerical computation. Indeed, the force $F_{m}^{*}$ at time $k+1$ is determined using information of positions and velocities at time $k$ (see figure 11):

$F_{m}^{*}(k+1)=B_{f p} \Delta V(k)+K_{f p} \Delta X(k)$ 
where:

$$
\begin{aligned}
& \Delta V(k)=A_{d} V_{s}^{*}(k)-V_{m}^{*}(k) \\
& \Delta X(k)=A_{d} X_{s}^{*}(k)-X_{m}^{*}(k)
\end{aligned}
$$

For similar reasons, the expression of $V_{s}^{*}$ is:

$V_{s}^{*}(k+1)=G_{n}\left[F_{e}^{*}(k)-\frac{1}{A_{f}} F_{m}^{*}(k)\right]$

Considering (32) and (34) and the fact that the position $X_{s}^{*}$ is computed using Tustin's discretization, when the probe is well above the substrate (no force applied on it, i.e. $\left.F_{e}^{*}=0\right), F_{m}^{*}$ is given by:

$F_{m}^{*}(k+1)=\lambda_{1} F_{m}^{*}(k-1)+\lambda_{2} V_{m}^{*}(k)+\left(\lambda_{3}+\lambda_{4}\right) K_{f p}$

where:

$\lambda_{1}=-G_{n}\left[\frac{B_{f p} A_{d}+K_{f p} A_{d} \frac{T_{e}}{2}}{A_{f}}\right]$

$\lambda_{2}=-\left[B_{f p}+K_{f p} \frac{T_{e}}{2}\right]$

$\lambda_{3}=A_{d}\left[X_{s}^{*}(k-1)+\frac{V_{s}^{*}(k-1) T_{e}}{2}\right]$

$\lambda_{4}=-\left[X_{m}^{*}(k-1)+\frac{V_{m}^{*}(k-1) T_{e}}{2}\right]$

Avoiding numerical instabilities leads to an upper bound on $G_{n}$, a necessary condition for stability:

$\left|\lambda_{1}\right|<1 \Leftrightarrow G_{n}<\frac{A_{f}}{A_{d} K_{f p} \frac{T_{e}}{2}+A_{d} B_{f p}}=G_{n_{l i m}}$

\subsubsection{Proportional integral controller $B_{f p}$ and $K_{f p}$}

In [27] a relation between $B_{f p}, K_{f p}$ and $T_{e}$ is derived to assure the stability of the system while in contact with a rigid environment. In that work, the authors apply the Routh-Hurwitz criterion to a control scheme similar to ours. However, the output of the coupling is the position $X_{m}$ instead of the velocity $V_{m}$ and the backward difference is used to determine the discrete controller $C(z)$ (instead of Tustin).

We will use the same methodology applied to our system. As for the DFF control scheme, the system considered is $L T I$.

The discrete time transfer function is:

$\frac{V_{m}^{*}}{F_{o p}^{*}}=\frac{V(z)}{1+V(z) C(z)}$

Before applying the Routh-Hurwitz criterion on the characteristic equation, a bilinear transformation ( $z=$ $\left.\frac{2+w T_{e}}{2-w T_{e}}\right)$ should be made. It leads to:

$b_{2} w^{2}+b_{1} w+b_{0}=0$ where:

$b_{2}=4 B_{v}(1+\delta)-4(1-\delta) B_{f p}$

$b_{1}=(1-\delta)\left(4 B_{v}+4 B_{f p}-2 K_{f p} T_{e}\right)$

$b_{0}=2 K_{f p} T_{e}(1-\delta)$

The Routh-Hurwitz criterion is achieved if and only if $b_{0}, b_{1}$ and $b_{2}$ have the same sign. Since $\delta<1$, (41) is always positive. Therefore, the system will be stable if and only if $b_{1}>0$ and $b_{2}>0$, which implies:

$$
\begin{gathered}
B_{v}+B_{f p}>\frac{K_{f p} T_{e}}{2} \\
B_{v} \frac{1+\delta}{1-\delta}>B_{f p}
\end{gathered}
$$

(42) is the same condition as that found in [27] and states that the stiffness of the coupling is bounded by the inherent damping of the haptic interface and that added by the coupling. Moreover, if the sampling period increases, $K_{f p}$ must decrease for the same amount of damping to guarantee stability.

Using the first order Taylor development of $x \mapsto$ $\exp (x)$ in the region of $0\left(\frac{B_{v} T_{e}}{M_{v}} \rightarrow 0\right.$ since $\left.T_{e} \rightarrow 0\right)$, $\delta \approx 1-\frac{B_{v} T_{e}}{M_{v}}$. (43) can be approximated by:

$\frac{2 M_{v}}{T_{e}}>B_{v}+B_{f p}$

This highlights that the maximum damping (and therefore, according to (42), the maximum stiffness) admissible is limited by the inertia of the master arm and is inversely proportional to the sampling period $T_{e}$. This is a convincing argument of the importance of the sampling period for stable haptic feedback.

\subsubsection{Summary}

The relations derived in section 4.3 as well as in paragraphs 4.4 .2 and 4.4 .3 are summarized in the table 2 . The minimum and maximum values each gain can take to ensure necessary stability conditions are given, as well as transparency considerations.

As seen in paragraph $4.4 .2, G_{n}$ has to be high to allow a transparent non contact behavior. According to (36), for stability reasons, $G_{n}$ must be smaller than $\frac{A_{f}}{A_{d} K_{f p} \frac{T_{e}}{2}+A_{d} B_{f p}}$. Therefore, smaller values for $B_{f p}$ and $K_{f p}$ allow for a higher $G_{n_{l i m}}$, and increase the non contact transparency.

Concerning $K_{f p}$, a maximum value to verify the Routh-Hurwitz criterion is derived. However, as explained above, for transparency reasons $K_{f p}$ should be low enough to limit the viscosity when there is no contact. 
Table 2 Valid range of values for $F P$ controller gains.

\begin{tabular}{|c|c|c|}
\hline & Minimum value & Maximum value \\
\hline$K_{f p}$ & $\begin{array}{l}\text { high for a stiff } \\
\text { contact feeling } \\
\text { (sec. } 4.3 .1)\end{array}$ & $(42),(44) \rightarrow \frac{4 M_{v}}{T_{e}^{2}}$ \\
\hline$B_{f p}$ & $(42) \rightarrow \frac{K_{f p} T_{e}}{2}-B_{v}$ & $\begin{array}{c}\text { low for non contact } \\
\text { transparency (sec. } \\
4.3 .2)\end{array}$ \\
\hline$G_{n}$ & $\begin{array}{c}\text { high for non } \\
\text { contact } \\
\text { transparency (sec. } \\
4.3 .2)\end{array}$ & $(36) \rightarrow \frac{A_{f}}{A_{d} K_{f p} \frac{T_{e}}{2}+A_{d} B_{f p}}$ \\
\hline
\end{tabular}

\subsection{Experimental results}

To compare the results with the ones obtained with the DFF control scheme we use the same cantilever as in section $3.5 .1\left(k_{c}=2.4 \mathrm{~N} \cdot \mathrm{m}^{-1}\right)$, with the same scaling factors $\left(A_{d}=0.05 \cdot 10^{6}, A_{f}=6 \cdot 10^{6}\right)$. Other parameters are chosen according to the results of the transparency and stability analysis (table 2).

A good compromise for transparency in contact and non contact mode is found if $K_{f p}=100 \mathrm{~N} \cdot \mathrm{m}^{-1}$. This value is indeed smaller than the maximum allowed value $\frac{4 M_{v}}{T_{e}^{2}}$. Enough damping is introduced by choosing $B_{f p}=$ $2.0 \mathrm{~N} \cdot \mathrm{s} \cdot \mathrm{m}^{-1}$. It is greater than the minimum value $\frac{K_{f p} T_{e}}{2}-B_{v}=0.15 \mathrm{~N} \cdot \mathrm{s} \cdot \mathrm{m}^{-1}$ and non contact transparency is still good. $G_{n}$ is chosen such that $G_{n}=$ $0.90 G_{n_{\text {lim }}}=48.0 \mathrm{~m} \cdot \mathrm{N}^{-1} \cdot \mathrm{s}^{-1}$.

The selected parameters must satisfy (21), (22) and (23) to ensure that the system will remain stable whatever the environment. In order to check such conditions, the values of $C_{1}, C_{2}$ and $C_{3}$ are plotted in the frequency domain. The results are given in figure 14.
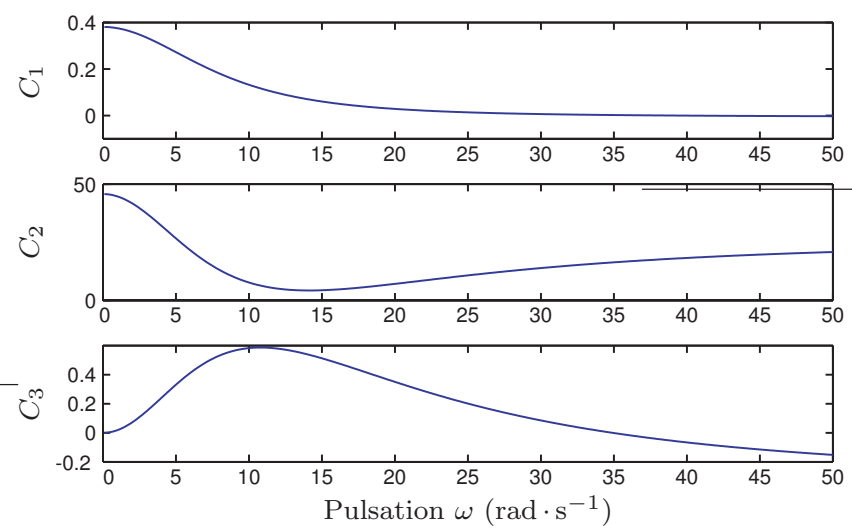

Fig. 14 Values of $C_{1}, C_{2}$ and $C_{3}$ with respect to $\omega$.

For pulsations lower than $\omega_{c}=35 \mathrm{rad} \cdot \mathrm{s}^{-1}, C_{1}, C_{2}$, and $C_{3}$ are positive: Llewelyn's criterion is verified. According to the system bandwidth, pulsations greater than $\omega_{c}$ will be attenuated, therefore the system will remain stable with the chosen values.

To verify experimentally that the gains we chose are adapted to the stated problem, we performed the same approach-retract operation as in section 3 . The results obtained are plotted in figure 15 . The forces felt by the operator, as well as those measured by the force sensor (and scaled by $A_{f}$ ) are represented. They must be compared to those in figure 9 . The system remained stable during this experiment, contrary to the DFF control scheme. Moreover, even if the force sent back to the user is computed through the control scheme (and not directly fed back), the feeling that the operator got reflects what happened in the remote environment since $F_{m}$ and $A_{f} F_{e}$ plots are similar. The oscillations that can be seen in $F_{m}$ 's plot were induced by the virtual coupling. Since the bandwidth of the haptic device is limited, they were not disturbing for the user. Therefore, although this control scheme is less transparent than the $D F F$, the feeling is good enough to allow the operator to feel the pull-off phenomenon with a peak of amplitude $1 \mathrm{~N}$. Consequently, the analysis performed in this paper allows for an efficient tuning of the controller presented in section 4.1 .

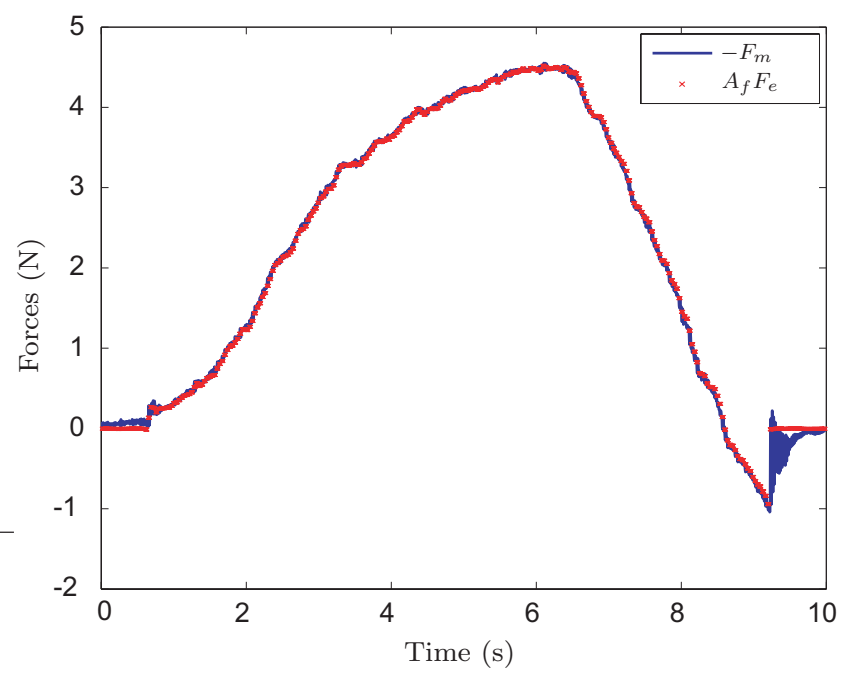

Fig. 15 Approach-retract curve for a cantilever of stiffness $k_{c}=$ $2.4 \mathrm{~N} \cdot \mathrm{m}^{-1}$, using $A_{d}=0.05 \cdot 10^{6}, A_{f}=6 \cdot 10^{6} . F P$ control scheme.

The pull-in phenomena is not visible on figure 15 . Indeed, to avoid time-consuming manipulations, we chose $A_{d}=0.05 \cdot 10^{6}$ (i.e., such that a velocity of $1 \mathrm{~cm} \cdot \mathrm{s}^{-1}$ of the haptic handle represents $0.2 \mu \mathrm{m} \cdot \mathrm{s}^{-1}$ for the slave device). With this value of $A_{d}$, the velocity of the nanotranslator was too high compared to the dynamics of 
the pull-in effect and thus could not be reflected to the user.

To prove the robustness of our approach with respect to the environment's stiffness, the same experiment was performed using different cantilevers. The results are presented in figure 16 . The same velocityscaling factor was used for the three probes. The force amplification was chosen so that the user could clearly feel the contact (about $5 \mathrm{~N}$ were fed back via the haptic device), for a cantilever's deflection smaller than $5 \mu \mathrm{m}$. This lead to $A_{f}=200 \cdot 10^{6}$ for the cantilever of stiffness $k_{c}=0.05 \mathrm{~N} \cdot \mathrm{m}^{-1}$, and $A_{f}=0.2 \cdot 10^{6}$ for stiffness $k_{c}=48 \mathrm{~N} \cdot \mathrm{m}^{-1}$. Other gains were chosen using table 2 .

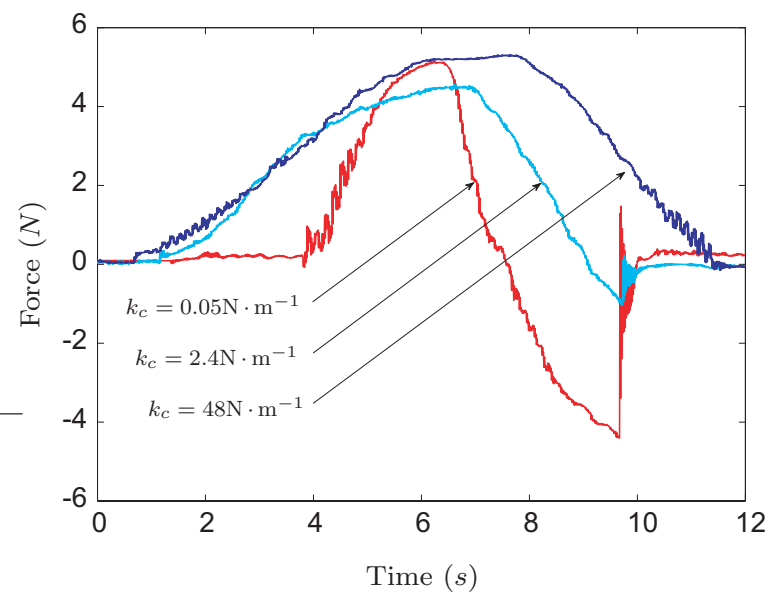

Fig. 16 Approach-retract curve for cantilevers of stiffnesses $k_{c}=0.05 \mathrm{~N} \cdot \mathrm{m}^{-1}\left(K_{f p}=100 \mathrm{~N} \cdot \mathrm{m}^{-1}, B_{f p}=1.2 \mathrm{~N} \cdot \mathrm{s} / \mathrm{m}\right.$, $\left.G_{n}=2329 \mathrm{~m} / \mathrm{N} \cdot \mathrm{s}\right), k_{c}=2.4 \mathrm{~N} \cdot \mathrm{m}^{-1}\left(K_{f p}=100 \mathrm{~N} \cdot \mathrm{m}^{-1}\right.$, $\left.B_{f p}=2.0 \mathrm{~N} \cdot \mathrm{s} \cdot \mathrm{m}^{-1}, G_{n}=48.0 \mathrm{~m} \cdot \mathrm{N}^{-1} \cdot \mathrm{s}^{-1}\right)$, and $k_{c}=$ $48 \mathrm{~N} \cdot \mathrm{m}^{-1}\left(K_{f p}=100 \mathrm{~N} \cdot \mathrm{m}^{-1}, B_{f p}=1.5 \mathrm{~N} \cdot \mathrm{s} \cdot \mathrm{m}^{-1}, G_{n}=\right.$ $\left.1.98 \mathrm{~m} \cdot \mathrm{N}^{-1} \cdot \mathrm{s}^{-1}\right)$. FP control scheme.

It should be noted that these experiments were performed in a non-controlled environment. Conditions of humidity and temperature may have changed between the experiments. However, the pull-off was indeed greater for cantilevers with a low stiffness. As noted above, the oscillations on the plots of figure 16 are induced by the virtual coupling, but are not disturbing for the operator due to the limited bandwidth of the haptic device.

The FP control scheme is adapted to nanomanipulations, especially because the system is stable, even when in contact with the substrate, for cantilevers with stiffnesses of $0.05 \mathrm{~N} \cdot \mathrm{m}^{-1}$ up to $48 \mathrm{~N} \cdot \mathrm{m}^{-1}$. Moreover, the analysis is validated experimentally. Forces of $10 \mathrm{nN}$ (pull-off for the cantilever of stiffness $k_{c}=0.05 \mathrm{~N} \cdot \mathrm{m}^{-1}$ ) were felt by the user.

\section{Conclusion}

Two different control schemes have been analysed in this paper, in the particular context of nanorobotic applications. The Direct Force Feedback control scheme suffers from a trade-off between stability and force amplification if time consuming manipulations have to be avoided. However, transparency is high as proven analytically, and underscored by the fact that we were able to feel both pull-in (forces of $0.5 \mathrm{nN}$ ) and pull-off phenomena. Using Force-Position control scheme, higher force amplification without suffering from the duration of the manipulation can be achieved. Forces of $10 \mathrm{nN}$ were felt by the user and stable contact for cantilevers of stiffnesses from $0.05 \mathrm{~N} \cdot \mathrm{m}^{-1}$ to $48 \mathrm{~N} \cdot \mathrm{m}^{-1}$ was demonstrated.

The choice of the control scheme will therefore depend on the relevant application. For a highly transparent rendering, $D F F$ is appropriate, however the experiments will be time-consuming. For a more complex task, implying high displacements, the FP control scheme should be chosen. It will greatly improve the operator's ability by providing him or her with force feedback.

Using the analysis carried out, the influence of each gain on the bilateral coupling is highlighted. These results will therefore help to realize real-time adaptation of the gains.

\section{Notations}

$$
\begin{array}{r|l}
F_{o p} / F_{e} / F_{m} & \text { User/environment/master force } \\
X_{s} / X_{m} & \text { Slave/master position } \\
V_{s} / V_{c} / V_{m} & \text { Slave/cantilever/master velocity } \\
Z_{o p} / Z_{e} & \text { User/environment impedance } \\
Z_{o p, L F}^{D F} / Z_{o p, H F}^{D F} & \text { Low/high frequencies user's impedance for DFF } \\
Z_{o p, L F}^{F P} / Z_{o p}^{F P}, H F & \text { Low/high frequencies user's impedance for FP } \\
k_{s} / k_{c} / k_{e q} & \text { Contact/cantilever/equivalent stiffness } \\
d & \text { Cantilever's deflection } \\
M_{v} / B_{v} & \text { Master's inertia/viscosity } \\
\tau_{i} & \text { i-th time constant of the nanotranslator } \\
V(s) / N(s) & \text { Virtuose/nanotranslator transfer function } \\
T_{e} & \text { Sampling period } \\
A_{f} / A_{d} & \text { Force/velocity scaling factor } \\
C(s) / C(z) & \text { Continuous/discrete PI controller } \\
B_{f p} / K_{f p} & \text { Proportional/integral gain } \\
G_{n} & \text { Proportional controller }
\end{array}
$$

Acknowledgements This work was supported by the French National Agency of Research, through the PACMAN project.

\section{References}

1. Sitti, M.: Microscale and nanoscale robotics systems [grand challenges of robotics]. IEEE Robotics and Automation Magazine 14(1), 53-60 (2007) 
2. Ferreira, A., Mavroidis, C.: Virtual reality and haptics for nanorobotics. IEEE Robotics and Automation Magazine 13(3), 78-92 (2006)

3. Hollis, R., Salcudean, S., Abraham, D.: Toward a telenanorobotic manipulation system with atomic scale force feedback and motion resolution. pp. 115-119 (1990)

4. Goldfarb, M.: Dimensional analysis and selective distorsion in scaled bilateral telemanipulation. In: Proceedings of the IEEE International Conference on Robotics and Automation, pp. 1609-1614 (1998)

5. Niemeyer, G., Slotine, J.J.: Stable adaptive teleoperation. IEEE Journal of Oceanic Engineering 16(1), 152-162 (1991)

6. Anderson, R., Spong, M.: Bilateral control of teleoperators with time delay. IEEE Transactions on Automatic Control 34(5), 494-501 (1989)

7. Ryu, J.H., Kim, Y.S., Hannaford, B.: Sampled- and continuous-time passivity and stability of virtual environment. IEEE Transactions on Robotics 20(4), 772-776 (2004)

8. Daunay, B., Micaelli, A., Régnier, S.: 6 DOF haptic feedback for molecular docking using wave variables. In: Proceedings of the IEEE International Conference on Robotics and Automation, pp. 840-845 (2007)

9. Kim, S.G., Sitti, M.: Task-based and stable telenanomanipulation in a nanoscale virtual environment. IEEE Transactions on Automation Science and Engineering 3(3), 240-247 (2006)

10. Boukhnifer, M., Ferreira, A.: H-infinity loop shaping bilateral controller for a two-fingered tele-micromanipulation system. IEEE Transactions on Control Systems Technology 15(5), 891-905 (2007)

11. Boukhnifer, M., Ferreira, A.: Wave-based passive control for transparent micro-teleoperation system. Robotics and Autonomous Systems 54(7), 601-615 (2006)

12. Kaneko, K., Tokashiki, H., Tanie, K., Komoriya, K.: Impedance shaping based on force feedback bilateral control in macro-micro teleoperation system. In: Proceedings of the IEEE International Conference on Robotics and Automation, pp. $710-717$ (1997)

13. Onal, C.D., Sitti, M.: A scaled bilateral control system for experimental one-dimensional teleoperated nanomanipulation. To be published in: International Journal of Robotics Research (2009)

14. Venture, G., Haliyo, D.S., Régnier, S., Micaelli, A.: Forcefeedback micromanipulation with unconditionally stable coupling. In: Proceedings of the IEEE/RSJ International Conference on Intelligent Robots and Systems, pp. 1923-1928 (2005)

15. Tian, X., Liu, L., Jiao, N., Wang, Y., Dong, Z., Xi, N.: 3D nano forces sensing for an AFM based nanomanipulator. In: Proceedings of the IEEE International Conference on Information Acquisition, pp. 208-212 (2004)

16. Xie, H., Vitard, J., Haliyo, D.S., Régnier, S., Boukallel, M.: Calibration of lateral force measurements in atomic force microscopy. Review of Scientific Instrument 79, 033,708 (6pp) (2008)

17. Onal, C.D., Pawashe, C., Sitti, M.: A scaled bilateral control system for experimental 1-D teleoperated nanomanipulation applications. In: Proceedings of the IEEE/RSJ International Conference on Intelligent Robots and Systems, pp. 483-488 (2007)

18. Ammi, M., Ferreira, A.: Robotic assisted micromanipulation system using virtual fixtures and metaphors. In: Proceedings of the IEEE International Conference on Robotics and Automation, pp. 454-460 (2007)

19. Ando, N., Ohta, M., Hashimoto, H.: Micro teleoperation with haptic interface. In: 26th Annual Conference of the IEEE Industrial Electronics Society, vol. 1, pp. 13-18 (2000)
20. Goethals, P., Gersem, G.D., Sette, M., Reynaerts, D., Brussel, H.V.: Accurate haptic teleoperation on soft tissues through slave friction compensation by impedance reflection. In: Proceedings of the Second Joint EuroHaptics Conference and Symposium on Haptic Interfaces for Virtual Environment and Teleoperator Systems (2007)

21. Maugis, D.: Contact, adhesion and rupture of elastic solids, vol. 130, chap. 4, pp. 203-344. Springer (2000)

22. Lawrence, D.: Stability and transparency in bilateral teleoperation. IEEE Transactions on Robotics and Automation 9(5), 624-637 (1993)

23. Hokayem, P.F., Spong, M.W.: Bilateral teleoperation: an historical survey. Automatica 42(12), 2035-2057 (2006)

24. Adams, R.J., Hannaford, B.: Stable haptic interaction with virtual environments. IEEE Transactions on Robotics and Automation 15(3), 465-474 (1999)

25. Hogan, N.: Controlling impedance at the man/machine interface. In: Proceedings of the IEEE International Conference on Robotics and Automation, pp. 1626-1631 (1989)

26. Llewellyn, F.: Some fundamental properties of transmission systems. Proceedings of the IRE 40(3), 271-283 (1952)

27. Gil, J.J., Avello, A., Rubio, A., Flórez, J.: Stability analysis of a 1 DOF haptic interface using the Routh-Hurwitz criterion. IEEE Transactions on Control Systems Technology 12(4), 583-588 (2004) 